\title{
LA INVESTIGACIÓN CIENTÍFICA Y SU VINCULACIÓN CON LA EDUCACIÓN SUPERIOR EN HONDURAS
}

\author{
Mario R. Acevedo Amaya, Universidad Nacional Autónoma de Honduras, \\ Instituto de Investigaciones Económicas y Sociales (IIESUNAH), \\ Ciudad universitaria edifico c2 primer piso. Tel./Fax: 504-22348973 \\ Correo electrónico: macevedo@iies-unah.org
}

\section{RESUMEN}

En un mundo totalmente globalizado, la investigación científica se ha convertido en el estandarte de los países denominados del primer mundo, las cuales basados en la triangulación de la Educación-Empresa Privada-Gobierno, conciben y desarrollan la ciencia e investigación. Los países del primer mundo descansan sus estrategias de crecimiento en la generación del conocimiento en la educación superior, y más aún la generación del conocimiento a partir de los Centros Universitarios Públicos. El gran éxito de esta estrategia radica en la voluntad política y disponibilidad de recursos para invertir en Investigación y Desarrollo (I/D), así como la participación activa de la empresa privada, ente que en los países industrializados estimula en un alto porcentaje el desarrollo de la ciencia, la innovación y la tecnología.

Ante un escenario mundial que evoluciona en los ámbitos de la ciencia y la investigación, es necesario conocer la situación actual y su porvenir. De manera más precisa, es necesario conocer sus cimientos, las cuales se originan desde la academia a través de la vinculación de la investigación científica con la educación superior, enfocándonos en la Universidad Nacional Autónoma de Honduras como medio trascendental para la ardua tarea de contribuir al desarrollo del país. Es por ello que el análisis de la correlación entre las variables de Educación superior y la investigación brindadas en el artículo dará a conocer la situación actual y contribuirá al afán de alcanzar que el sistema de educación superior de paso a la investigación científica como parte del diseño curricular para el desarrollo de insumos con conocimientos y competencias de clase mundial.

Palabras clave: Generación del Conocimiento, Estrategias de Crecimiento, La Educación Superior, La Investigación Científica. 


\title{
SCIENTIFIC RESEARCH AND ITS RELA- TIONSHIP WITH HIGHER EDUCATION IN HONDURAS
}

\author{
Mario R. Acevedo, Universidad Nacional Autónoma de Honduras, \\ Instituto de Investigaciones Económicas y Sociales (IIESUNAH), \\ Ciudad universitaria edifico c2 primer piso. Tel./Fax: 504-22348973 \\ Correo electrónico: macevedo@iies-unah.org
}

$\mathbf{E} \& \mathbf{A}$ IIES

\section{ABSTRACT}

In a completely globalized world, the scientific research has become the standard of the well know world's first class, which based on the triangulation of the Education-private organizations-government, that conceives and develops the know/how of the scientific research.

The first world class countries relay their growth strategies upon the knowledge generated at the university level, and the knowledge emitted by the public educational institutions. The strategies' success is directly influenced by the political forces and the level of resources available to invest in, particularly in Research and Development activities (R/D), as well as the proactive participation of the companies that are directly involved. Therefore, the entities that interact across the industrialized countries, tend to stimulate the scientific, technological and innovative development of specific academic areas.

By Living in such an unstable world that is characterize for the constant facing of extreme conditions, it has become necessary to comprehend the current and future situation by accurately forecasting its reality; in order to contextualize the scientific research in the region. Therefore, is essential to know their basis, which derives from the academy through the bonding of the scientific research and higher education programs, focusing in the Universidad Nacional Autónoma de Honduras, as a way to transcend the difficult work that awaits the national development. Thus, the correlation analysis between higher education and scientific research, expects to facilitate and release the current situation, contributing in the achievement of the goals that are presently pursuit by the higher education system which includes knowledge generation as a part of the curricular design for the development of the world class inputs skills and knowledge.

Keywords: Knowledge Generation, Growth Strategies, Higher Education, Scientific Research. 


\section{INTRODUCCIÓN}

El comienzo de un nuevo siglo se convierte en un espacio de múltiples y aceleradas transformaciones en ámbitos económicos, sociales, tecnológicos y políticos. En el contexto internacional obedecemos a un reordenamiento general con cambios vertiginosos y fundamentales en los campos de la producción, la cultura y la organización social. En tiempos en que la revolución tecnológica e informática y sus efectos en el trabajo y la cultura, así como la globalización y el intercambio de mercados globales controlan las economías, nos invita a estudiar su concepción basada a partir del uso intensivo del conocimiento como fuente para la creación de nuevos modelos de desarrollo productivo y económico (Drucker, Los Desafíos de la Gerencia en el Siglo XXI, 2002).

A su vez, en un mundo globalizado, el crecimiento de los países descansa en gran medida, en el desarrollo de los conocimientos y aportes en materia de investigación científica originadas desde el sector educativo. Por lo que es de carácter relevante efectuar un análisis de las actividades y preparación en materia de investigación realizadas a nivel mundial, a fin de contextualizar en este ámbito al país. La medición de las actividades en Investigación, Desarrollo e Innovación $(I+D+i)$, así como las competencias del personal que transfiere los conocimientos científicos a la población estudiantil nos permitirá conocer la situación actual del país para crear una consciencia crítica de las actividades en materia de desarrollo de la ciencia, investigación y la innovación que se están generando desde cada sector y en especial desde la academia.

E\&A

IIES

83

La necesidad de conocer la situación actual de Honduras en cuanto a inversiones en $\mathrm{I} / \mathrm{D}$, y en especial el papel que está jugando la academia como ente que enarbola la transferencia de conocimientos científicos, da origen a la siguiente hipótesis: en un escenario ideal, A mayor número de docentes en la Universidad Nacional Autónoma de Honduras (UNAH) y fundamentados en que es el ente que rectora la educación del país, mayor deberán ser los docentes identificados como investigadores, y mayor aun el numero de los docentes que actualmente están haciendo investigación. Dicha aseveración ha de ser valida si el porcentaje de docentes bajo el supuesto es representativo en relación a su masa crítica total. El porcentaje se considerará representativo si éste supera en más de la mitad a la población docente universitaria.

\section{MARCO DE REFERENCIA}

En un contexto cuyo centro de gravedad de las principales fuentes de valor agregado se ha reorientado al recurso intelectual como insumo crítico para la producción de riqueza, convierte el acceso al conocimiento en el ámbito científico en una ventaja comparativa para los individuos, las empresas y 
economías nacionales, siempre que estos conviertan los mismos en tecnología, productividad, desarrollo organizacional y en consumos sostenibles y racionales (Contigiani, 2001). Lo antes citado es conocido como "Economía basada en el conocimiento o economía digital" surgida a partir de la instauración de un orden mundial telemático-comunicacional, que abarcó desde las MIPYMES hasta los grandes grupos económicos (Mangioni, 2011).

Por otra parte, las amplias nociones de economía basada en el "conocimiento", "sociedad del conocimiento" y "sociedad de aprendizaje", describen un modelo ideal de producción y cultura (Mateo, 2006). Sin embargo, aún en el mundo desarrollado, esta transformación no escapa a fuertes tensiones y resistencias, entre las cuales cabe referir; las amplias tendencias a la polarización desencadenadas por la injusta distribución de las oportunidades educativas, la confrontación entre la lógica de la producción de conocimientos en los centros académicos versus su apropiación y uso en las empresas, la presión sobre las universidades en torno a sus ofertas curriculares y agendas de investigación, las tendencias a la privatización de las instituciones de enseñanza superior cuando son vistas en primer lugar como proveedoras de bienes cotizados en el mercado, entre otras (Contigiani, 2001).

A pesar de ello, debemos tener presente que la noción de sociedad del conocimiento es ante todo una expresión valorativa, no una expresión que cumpla propósitos descriptivos, analíticos o explicativos. Pero precisamente por su carácter utópico orientado a cambios de diversas esferas de la realidad, más bien precisa a que diversas innovaciones originadas en los campos de la producción, la tecnología, la ciencia y la cultura converjan hacia la definición de políticas públicas (Drucker, La sociedad post-capitalista, 1999). De modo que nuevas exigencias, demandas y oportunidades surgen ante los sistemas de educación superior, investigación científica y tecnológica, debido a su papel clave en la generación y movilización de conocimientos, así como por sus posibilidades de formación de personas con capacidades de desempeño creativo y de adaptación a los cambios.

Dichos cambios exigen nuevas estrategias enmarcadas en la modernización y adecuación de sistemas del conocimiento en la educación, entre las cuales cabe destacar: expansión de la cobertura y oferta educativa; creación de instancias de regulación y coordinación, vinculación con la ciencia y la investigación, implantación de fórmulas de planeación, evaluación y rendimiento de cuentas, mecanismos de aseguramiento de la calidad, flexibilidad curricular, incorporación de formas de aprendizaje a distancia y diseño de esquemas para la actualización de conocimientos y renovación de destrezas (educación para toda la vida), entre otras (Contigiani, 2001).

Las nuevas estrategias de modernización del sistema educativo y la propia dinámica de los sistemas de educación superior, ciencia y tecnología traen 
consigo nuevas líneas de interrelación; estas dinámicas tienden a apoyarse mutuamente y crear sinergia a través de medios estratégicos tales como los pregrados, postgrados de investigación, convenios de vinculación, los programas gubernamentales de fomento y los fondos privados para el financiamiento de los proyectos. No obstante, hoy en día nos enfrentamos a dos amplias tendencias, la primera de ellas: "la separación del desarrollo científico y las universidades", la cual se explica por motivos de especialización funcional, aunque también por la acción de fuerzas de mercado. La segunda tendencia se caracteriza por la "pérdida de atributos de la universidad" (Newman, 2000), dando origen a una nueva directriz hacia la formación de varios circuitos, relativamente autónomos de generación, difusión y aprovechamiento del conocimiento.

\subsection{Las Inversiones en I/D en el nuevo Mundo}

En los países con economías más sólidas, la prioridad concedida a la educación superior y a la investigación científica es reflejada en una nueva oleada de expansión de la matrícula universitaria, y en un crecimiento significativo de la inversión pública y privada en actividades de investigación y desarrollo (I/D) (Huergo, 2006). En la década de los noventa la tasa de cobertura de la demanda potencial (representada por el grupo de edad entre 20 y 24 años) creció en esos países del $45 \%$ al $60 \%$. Incluso en Europa occidental se ha registrado lo que algunos observadores denominan un "nuevo ciclo de masificación" de la educación universitaria. En tanto, en América Latina la demanda cubierta pasó del $16 \%$ al $20 \%$ durante el mismo período, crecimiento que se explica principalmente por una sostenida expansión del grupo de instituciones privadas (Contigiani, 2001).

El contraste entre la expansión en la cobertura por aumento de la matrícula universitaria en los países desarrollados versus los subdesarrollados, se encuentra en la capacidad y el gasto en I/D que realiza cada uno de ellos. Disparidad que resulta neurálgico y que continua siendo el talón de Aquiles de las políticas de innovación en ciencia y tecnología (ITC) en los países de América Latina, a excepción de Brasil que representa por si solo el $60 \%$ del Gasto en I+D del conjunto de la región (UNESCO, 2010). En 2007, el gasto bruto en investigación y desarrollo (GBID) de América Latina y el Caribe alcanzó apenas un $0,67 \%$ del Producto Interno Bruto (PIB), esto es un 3\% del gasto mundial en I+D, si se convierte en dólares a paridad de poder adquisitivo. En los países de la Organización para la Cooperación y el Desarrollo Económico (OECD), el promedio del GBID con respecto al PIB asciende a un 2,28\%, por lo que no es sorprendente que las personas dedicadas a la investigación en la región representasen solamente el 3,5\% del número mundial total de investigadores al 2007. Si bien es cierto que ese porcentaje había experimentado un aumento desde el 2002, año en el que se cifró en un 2,9\%, cabe señalar que casi la mitad 
del porcentaje en cuestión (1,7\%) correspondió a Brasil (UNESCO, 2010).

Las diferencias a nivel mundial en el campo de la Investigación y Desarrollo son abismales, mientras los países desarrollados tienen un crecimiento (en miles de personas) del 2002 al 2007 de 4,047.5 a 4,478.3 investigadores, los países en desarrollo sufren un cambio de 1,734.4 a 2,696.7 en 2007; resulta más notable las estadísticas de los países menos adelantados, el cual se sitúan con un aumento del 2002 al 2007 de 28.7 a 34.7 investigadores. Otro aspecto importante que requiere mención, es la distribución de los investigadores por cada millón de habitantes, el cual en países desarrollados es de 3,655.8, en países en desarrollo 580.30, y en los países menos adelantados es de 43.4 investigadores por cada millón de habitantes (UNESCO, 2010).

Ante tal situación, algunos países de la América Latina se han interesado y han re direccionado todos sus esfuerzos y políticas a aquellas que propicien la innovación, tal es el caso de Argentina, Brasil, México y Chile que concentran el $90 \%$ de las inversiones en I+D en Latinoamérica. Sin embargo, en América Latina la inversión I+D depende en un $60 \%$ de los recursos financieros proporcionados por el Estado, de los cuales el $40 \%$ son canalizados a través de sus dependencias a los centros Universitarios de alto rendimiento y la diferencia a instituciones públicas de investigación. A pesar de ello, los esfuerzo aún siguen siendo cortos para el resto de países de América Latina que evidencian el poco crecimiento en el campo de la ciencia y la investigación, ante la ausencia de políticas de gobierno y educación enmarcada en la inversión en I/D (UNESCO, 2010).

\section{ANÁLISIS DE LA INVERSIÓN Y DESARROLLO EN I/D EN LA EDUCACIÓN SUPERIOR EN HONDURAS}

\subsection{Contexto en la Universidad Nacional Autónoma de Honduras}

Honduras ha logrado un comienzo vinculador, a través de la inclusión de la inversión en I/D como pilar fundamental del Plan de Nación, la cual mediante la organización e interacción de la cuádruple hélice Gobierno, Sociedad, Academia y Empresa Privada pretende crear sinergia y sumar todos sus esfuerzos para el desarrollo de la ciencia y la investigación desde la educación superior (Trejos, 2007). La inversión en I/D que realiza el gobierno de Honduras hoy en día ronda el $0.0013 \%$ del PIB (IntraMed, 2007), dicha inversión en I/D es inferior en comparación a países de la región como Panamá que invierte el $0.21 \%$ de su PIB, Costa Rica 0.4\%, El Salvador 0.09\%, Guatemala 0.06\% (Banco Mundial, 2006-2010), Nicaragua 0.05\% (Gabriel Mayaca Trejos, 2010). El bajo porcentaje del PIB destinado a I/D nos rezaga en el ámbito de inversiones en I/D en Centroamérica. 
Para alcanzar estándares de clase mundial en la I/D y contar con al menos el $2 \%$ del PIB invertido en I/D tal como lo hacen los países desarrollados, el Estado de Honduras en conjunto con la empresa privada, deberán invertir al menos 20 veces el valor que actualmente se utiliza. La cifra rondaría los \$245, $000,000.00$, labor compleja pero necesaria si se quiere posicionar en el concierto mundial como un país que invierte de forma constante en la investigación y desarrollo (Consejo Hondureño de Ciencia y Tecnologia, 2011), para ello, será necesario involucrar de manera directa y vinculante a la empresa privada con el gobierno, para simular los escenarios de los países desarrollados tales como Japón, en el que el sector privado contribuye con el 80\% (Consejo Hondureño de Ciencia y Tecnologia, 2011) de la inversión en I/D (Barquero, 2003).

En el país, la Universidad Nacional Autónoma de Honduras (UNAH) es la encargada de revertir la situación actual en cuanto a I/D, a través de la generación de la investigación, la innovación y ciencia para el desarrollo nacional, no por imposición sino por su fin mismo. Ante tal situación, la UNAH ha dispuesto recursos para que la creación de una Dirección de Investigación Científica la cual es la instancia academia que debe coordinar las actividades de innovación, investigación y desarrollo de la ciencia. Al pasar de los años, los recursos financieros de la Dirección de Investigación Científica se han visto limitado al $1 \%$ del presupuesto de la UNAH (Comisión de Control de Gestión UNAH, 2011), valor condicionado en relación a la cantidad de trabajo que se pretende desarrollar en esta materia.

Sin embargo, en la actualidad la Dirección de Investigación Científica (DICU) ha asumido un nuevo enfoque para encarar la innovación, investigación científica y tecnológica dentro de la institución. Se plantea la apertura y restructuración de las unidades de Investigación de la UNAH, con el registro de catorce Unidades de Investigación, la cual están de forma constante llevando a cabo actividades para la especialización del personal docente en los campos de la investigación, así como para apoyar y estimular al alumnado a adentrarse en dicho ámbito (Universitaria, 2007). No obstante, la creación de unidades y restructuración de las actuales no ha permitido contar con el acompañamiento de todos los docentes en la UNAH, ya sea por ausencia o no cumplimiento de las políticas o reglamentos que exigen la inclusión de la investigación científica en los programas académicos o bien por no exigir al personal docente su incorporación para su formación y la del estudiante en los campos de la ciencia y la investigación. Es por ello que en el país la vinculación entre la investigación científica y la educación superior se ve sumida en una amplia brecha que imposibilita el desarrollo de la I/D en el país.

A fin de conocer la vinculación de la Educación Superior y la Investigación Científica, a través del número de docentes involucrados en esta área, se procedió a analizar, en base a los datos estadísticos de fuentes secundarias, la 
situación actual del personal docente que labora para la UNAH. De un total de 3,343 docentes se encuentran involucrados de manera activa 86 docentes como investigadores, porcentaje muy discreto del $2.57 \%$ del total de la masa crítica (Cientifica, 2010), diseminados los más representativos en las áreas que a continuación se detalla:

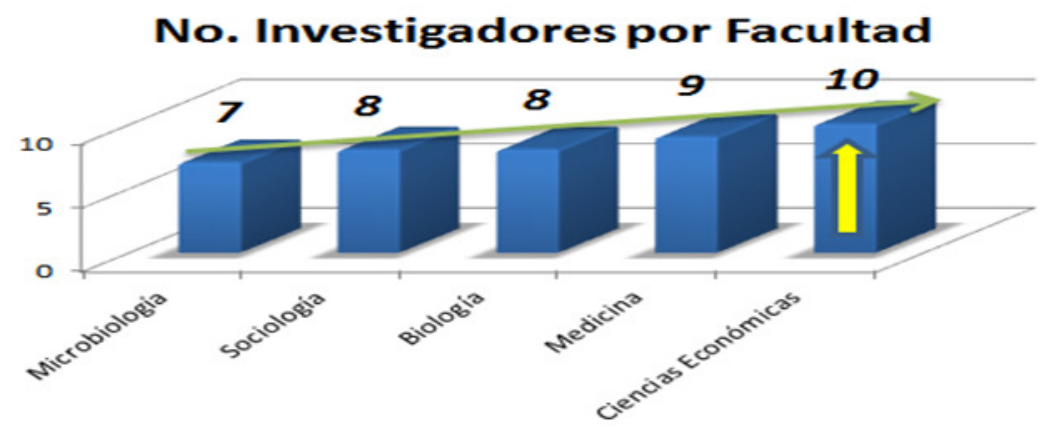

Gráfico 1. Número de investigadores por facultad (Fuente: Memoria de UNAH 2010)

$\mathbf{E} \& \mathbf{A}$ IIES 
Al analizar las variables docente por facultad y docentes por facultad adscritos a la DICU, es decir que realizan investigación de manera formal, la facultad que tiene una mayor presencia en el desarrollo de la investigación es la de Microbiología, esto a razón que un alto porcentaje de su masa crítica de docentes está involucrado en la investigación, y desvirtúa el dato estadístico que a simple vista nos refleja a la Facultad de Ciencias Económicas como la que cuenta con el mayor número de investigadores, ya que solo el 3\% de sus docentes dedican esfuerzos a tal labor (Direccion de Investigacion Cientifica, 2009).

Es por ello que el gráfico No. 2 refleja que: “A mayor número de catedráticos, menor es el número de investigadores", a pesar que la relación según la lógica, debe ser: "A mayor catedráticos, Mayor número de investigadores". Revelándonos el análisis que la relación docente por Facultad-Investigadores es inversamente proporcional en todas sus facultades, esto según los datos proporcionados por la Memoria UNAH 2010 y el catálogo de investigadores de la DICU.

Otro aspecto fundamental a analizar en el ámbito del desarrollo de la ciencia y la investigación desde la UNAH, es la cantidad de estudiantes por docente universitario y más importante aún la cantidad de docentes universitarios investigadores por estudiante. Para el análisis se consideró la población de 70,303 estudiantes y un total de 3,343 docentes con que cuenta la UNAH. Al tomar dichos datos y realizar la correlación entre las variables estudiante-docente, así como las variables estudiante-docente-investigador, se determinó que existen al menos 21 estudiantes por catedrático universitario, esto de forma global. Un excelente número en comparación al estándar de países como Estados Unidos de Norte América, la cual cifran en 24 estudiantes como número óptimo de estudiantes por docente (Candela, 2002) ${ }^{1}$.

No. de Estudiantes por Docente UNAH

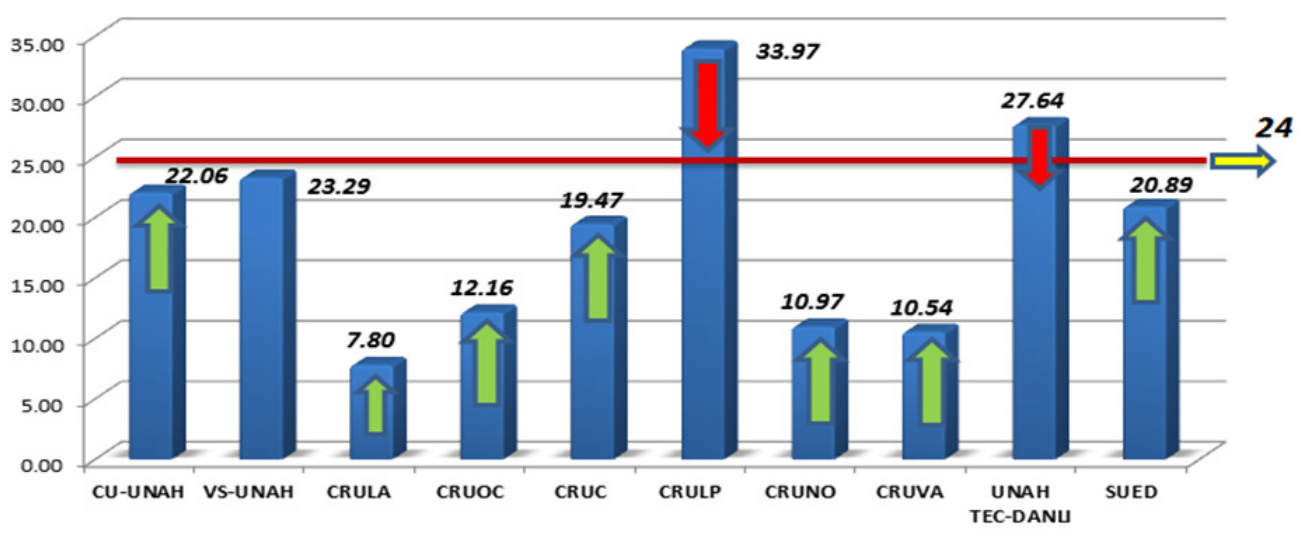

Gráfico 3. Relación docentes versus estudiantes por Centro Universitario

(Fuente: Memoria de UNAH 2010, Docentes por Centro Universitario y Matrícula por Centro Universitario 2010) 
Aunque el número de estudiantes por docente es óptimo en la mayoría de los centros universitarios de la UNAH, al analizar la correlación docenteinvestigador por estudiante, y bajo la considerando de que existen tan solo 86 docentes investigadores adscritos a la DICU, nos revela la existencia de al menos 1 catedrático investigador por cada $817^{2}$ alumnos. Lo anterior nos lleva a concluir que existe una ausencia marcada del docente en la incursión en el campo de la investigación científica y la mínima posibilidad que los estudiantes sean educados bajo líneas de investigación científica. Los datos estadísticos del 2010 antes mencionados y extraídos de las memorias de la UNAH, nos comprueban la amplia brecha que tiene el país en el desarrollo de la ciencia y la investigación desde la educación superior, lo que nos ubica en el contexto internacional como un país que no apunta todos sus esfuerzos a la generación I/D como polo de desarrollo y crecimiento de la nación.

En este sentido, ante un panorama retador para los que enarbolan las banderas de la investigación al interior de la UNAH, así como para las autoridades de la academia, será necesario dictar pautas para que cada una de las unidades de investigación en todas las Facultades de la UNAH se den a la tarea de capturar un mayor números de docentes para insertarlos al ámbito de la investigación, y estos a su vez puedan trasladar los conocimiento y más aun el incentivo por realizar investigación en el estudiante, para que desde las aulas de clases se desarrollen los conocimientos científicos requeridos por el mercado demandante.

\section{A MANERA DE PROPUESTA}

La situación actual empieza a motivar a las Facultades de la UNAH, tal es el caso de la Facultad de Ciencias Económicas a través del Instituto de Investigaciones Económicas y Sociales (IIES) que consideran que en la educación superior existe una oportunidad para abanderar el cambio a través de un nuevo modelo de vinculación de la ciencia y la investigación con la academia, enfocada en primera instancia a la masa crítica docente y estudiantil del Sistema de Posgrado de la Facultad de Ciencias Económicas (POSFACE) y en segunda instancia a la población de la Facultad de Ciencias Económicas (FCE). La vinculación consistirá en la articulación de los docentes con los investigadores del IIES, a través de sus cuatro grandes proyectos; las Incubadoras de Ciencia y de Emprendimiento, Producción de alto Rendimiento en Bienes y Servicios, El Observatorio Económico y de Emprendimiento (OEE) y el Observatorio de Operaciones.

Se pretende realizar la articulación en dos vías; la primera, con la preparación del docente en el área de la investigación científica, y la inclusión para todos de una asignatura cuyo fin primordial será el desarrollo de los fundamentos de la Calidad y la Innovación. La segunda, será mediante la participación activa 
de las asignaturas con relación directa o indirecta bajo las líneas de investigación de la FCE. Dicha articulación estratégica proporcionará información tanto primaria como secundaria, a lo interno y externo del centro de estudio. La información que de ahí surjan tendrá como resultado la generación de ideas plasmadas en proyectos de investigación, que serán llevados a las Incubadoras de Ciencia para su estudio científico, así como para el análisis de su viabilidad y factibilidad. Si las antes mencionadas resultan ser redituables en aportes teóricos como financieros, se enviarán a la Incubadora de Emprendimiento para su desarrollo. Con la información obtenida se retroalimentaran las bases de datos de los Observatorios, a fin de ser un brazo extendido en la ardua tarea de actualización y comprobación de datos en el campo de acción, permitiendo a su vez la búsqueda y desarrollo de nuevos modelos, componentes, prototipos o innovaciones, que vendrán a revolucionar y a aportar significativamente a la industria de bienes y servicios. Todo esto se deberá realizar sin menoscabo de la propiedad intelectual que del mismo emane.

El modelo citado permitirá dar un primer paso e involucrará a los docentes con una mayor formación, a enrolarse en el campo de la investigación científica como un primer paso para luego ser replicado al sistema de pregrado de la UNAH. El resultado se fundamentara en el aumento de los aportes para el desarrollo de la I/D desde la academia, motivando a los demás sectores a incluirse mediante la inversión en ciencia y tecnología en pro del desarrollo de la nación.

\section{CONCLUSIONES}

A manera de concluir, las pautas indicadas han comenzado a modificarse en los últimos años, a raíz de los amplios debates mundiales sobre el valor estratégico del conocimiento, por lo que se vislumbra en el horizonte un sinfín de consensos sobre la necesidad de transformar los sistemas de educación superior, ciencia y tecnología, en el sentido de enriquecer su capacidad de respuesta a los requerimientos del entorno inmediato y la dinámica de la globalización.

A su vez, los resultados de la investigación nos muestran la brecha que existe entre la docencia y la investigación, revelándonos que de un total de 3343 docentes que laboran en la UNAH solo 86 de ellos se encuentran formalmente adscritos a la DICU y están realizando investigación científica de forma constante, incitándonos a incentivar a los docentes a participar en este ámbito para que desde la máxima casa de estudios del país se genere el cambio y el desarrollo de I/D para el país.

Será tarea diaria de los investigadores pertenecientes a las diferentes disciplinas y áreas del conocimiento miembros de los Institutos de Investigación Universitarios, así como a todos aquellos investigadores científicos de otras 
instituciones afines contribuir para que mediante esa reforma, el país se integre al ámbito internacional en el campo de la investigación científica, como parte fundamental de la educación superior y nos permita situarnos en una posición importante en cuanto a ciencia y desarrollo tecnológico se refiera.

\section{LIMITACIONES Y FUTURAS INVESTIGACIONES}

Al indagar e investigar acerca de las inversiones y acciones en materia de Innovación y Desarrollo (I/D) que ha realizado el país a través del tiempo, desde los distintos sectores educativos, privados y gubernamentales, los investigadores se encuentran con un sinfín de barreras que imposibilitan obtener información precisa acerca de las actividades que se están desarrollando y otras que se encuentran por desarrollar, lo que hace más tediosa la generación de investigaciones en un tema tan sensible y de carácter prioritario para el país. Por otro lado, existe un vacío de información acerca de la Educación Superior, investigación científica, desarrollo e innovación realizadas desde la academia, lo que impide crear un punto de partida y un marco de referencia sustentado y validado por los aportes que puedan surgir desde las unidades encargadas de su estudio. A su vez, el investigador se encuentra con la desactualización de la mayoría de las bases de datos e indicadores del país, incluyendo las generadas desde la academia. Las bases de datos de la UNAH no es la excepción y los datos de

E\&A IIES los docentes investigadores no se escapan a tal realidad, fomentando un sin número de discrepancias en la veracidad de la información secundaria solicitada para el desarrollo de las investigaciones en este ámbito.

Todo lo anterior se resume en la falta de interés de las autoridades, unidades académicas, gobierno, empresa privada y docentes, a abordar esta temática en el país. A pesar de ello, dichas falencias dan origen a futuras investigaciones en materia de desarrollo e innovación en todos los ámbitos, desde el empresarial hasta el académico. Por lo que se requiere la suma de los esfuerzos para crear sistemas integrados de investigadores e investigaciones que tengan el único fin de desarrollar la ciencia del conocimiento y ser pilar fundamental para el progreso de la nación.

\section{RECONOCIMIENTOS}

Esta investigación ha sido financiada parcialmente por el Consejo Nacional de Educación de Honduras, la Beca Sustantiva de Investigación No. 03-2011 por parte de la Dirección de Investigación Científica y por la Vice-rectoría académica de la UNAH. El autor desea agradecer el apoyo del Gobierno de Honduras y de la UNAH. 


\section{REFERENCIAS}

Banco Mundial. (2006-2010). El Banco Mundial/ Indicadores. Recuperado el 18 de 10 de 2011, de http://datos.bancomundial.org/indicador/ GB.XPD.RSDV.GD.ZS

Barquero, I. (Noviembre de 2003). http://www.undp.un.hn. Recuperado el 16 de Octubre de 2011, de http://www.undp.un.hn/publicaciones/colecciones/ DH_21.pdf

Candela, A. (02 de 06 de 2002). e-valencia.org. Recuperado el 17 de 10 de 2011, de http://e-valencia.org/index.php? name $=$ News $\&$ file $=$ article $\&$ sid $=1119$

Direccion de Investigacion Cientifica. (2010). Memoria UNAH 2010. Tegicugalpa, Honduras: Editorial Universitaria.

Comisión de Control de Gestión UNAH. (20 de 01 de 2011). https:// www.unah.edu.hn. Obtenido de https://www.unah.edu.hn: https:// www.unah.edu.hn/uploaded/content/category/1742185874.pdf

Consejo Hondureño de Ciencia y Tecnologia. (20 de Septiembre de 2011). www.gob.hn. Recuperado el 22 de Octubre de 2011, de http://www.gob.hn/ portal/poder_ejecutivo/consejos_y_comisiones/cohcit/

Contigiani, R. R. (2001). Desarrollo de las Ciencias Sociales y las Humanidades en Mexico. Mexico: Editorial UNAM.

Dirección de Investigación Cientifica. (2009). Catalogo de Investigadores. Tegucigalpa, Honduras: Editorial Universitaria.

Drucker, P. F. (1999). La sociedad post-capitalista. Bogota: Editorial Norma.

Drucker, P. F. (2002). Los Desafios de la Gerencia en el Siglo XXI. New York, Estados Unidos: Grupo Editorial Norma.

Gabriel Mayaca Trejos. (2010). El Rol de las Universidades en el Desarollo Científico-Tecnológico. Managua, Nicaragua.

Huergo, M. S. (Octubre de 2006). http://info.uned.es. Recuperado el 14 de Octubre de 2011, de http://info.uned.es/experto-universitario-gestion-I-D/ bibliografia/bibliografia\%202/BIBLIOGRAFIA/LA\% 20FINANCIACION\%20DE\%20LAS\%20ACTIVIDADES\%20DE\% 20I+D+I_CDTI.pdf

IntraMed. (13 de Jun de 2007). Ciencia en Centro América.

Mangioni, J. C. (2011). Economia basada en el Conocimiento. Brasil , Brasil.

Mateo, J. L. (2006). Sociedad del conocimiento. ARBOR Ciencia Pensamiento y Cultura, 145-151. 
Trejos, G. M. (2007). http://www.cinda.cl. Recuperado el 24 de Octubre de 2011, de http://www.cinda.cl/download/informes_nacionales/ honduras10.pdf

UNESCO. (2010). Informe de la Unesco Sobre la Ciencia. Francia: Ediciones Unesco.

Dirección de Investigación Científica. d. (2007). La Dirección de Investigación Científica: Las bases del Sisitema de Investigacion de la Universidad Nacional Autonoma de Honduras. Tegucigalpa, Honduras. 